Symmetry and Physical Properties of Crystals 


\section{Grenoble Sciences}

The aim of Grenoble Sciences is twofold:

- to produce works corresponding to a clearly defined project, without the constraints of trends nor curriculum,

- to ensure the utmost scientific and pedagogic quality of the selected works: each project is selected by Grenoble Sciences with the help of anonymous referees. In order to optimize the work, the authors interact for a year (on average) with the members of a reading committee, whose names figure in the front pages of the work, which is then co-published with the most suitable publishing partner.

Contact: Tel.: (33) 476514695

E-mail: grenoble.sciences@ujf-grenoble.fr

Website: https://grenoble-sciences.ujf-grenoble.fr

Scientific Director of Grenoble Sciences

Jean Bornarel, Emeritus Professor

at the Joseph Fourier University, Grenoble, France

Grenoble Sciences is a department of the Joseph Fourier University

supported by the ministère de l'Enseignement supérieur

et de la Recherche and the région Rhône-Alpes.

Symmetry and Physical Properties of Crystals is an improved version of the original book Symétrie et propriétés physiques des cristaux by Cécile Malgrange, Christian Ricolleau and Françoise Lefaucheux, EDP Sciences, Savoirs Actuels Series, 2011, ISBN 9782759804993.

The Reading Committee included the following members:

- Ryszard Cach, Professor, University of Wrocław (Wrocław, Poland)

- Vladimir E. Dmitrienko, Chief Researcher, A.V. Shubnikov Institute of Crystallography (Moscow, Russia)

- José Goulon, Emeritus CNRS Senior Researcher, former group leader at the European Synchrotron Radiation Facility (Grenoble, France)

- Hiroo Hashizume, Emeritus Professor, Tokyo Institute of Technology (Tokyo, Japan)

- Jochen Schneider, Professor, former DESY Research Director (Hamburg, Germany), former director of the Experimental Facilities Division for the LCLS (Stanford, USA)

Translation from original French version performed by Michel Schlenker; typesetting: Stéphanie Trine-special thanks to Konstantin Protassov and Alexei Voronine; figures: Sylvie Bordage, Alain Jeanne-Michaud; cover illustration: Alice Giraud, with sample of pyrite: mineral collection of Pierre and Marie Curie University, photograph Jean-Pierre Boisseau 
Cécile Malgrange $\cdot$ Christian Ricolleau Michel Schlenker

\section{Symmetry and Physical Properties of Crystals}

Springer 
Cécile Malgrange

Université Pierre et Marie Curie

Institut de Minéralogie, de Physique des

Matériaux et de Cosmochimie

case 115,4 place Jussieu

75252 Paris cedex 05

France

Christian Ricolleau

Université Paris Diderot-Paris 7

Laboratoire Matériaux et Phénomènes

Quantiques

Bâtiment Condorcet, case 7021

75205 Paris cedex 13

France
Michel Schlenker

Université Grenoble-Alpes

Grenoble-INP et Institut Néel du CNRS

B.P. 166

38042 Grenoble cedex

France

Translated, revised and adapted from "Symétrie et Propriétés Physiques des Cristaux", Cécile Malgrange, Christian Ricolleau et Françoise Lefaucheux, EDP Sciences/CNRS Éditions 2011

ISBN 978-94-017-8992-9

ISBN 978-94-017-8993-6 (eBook)

DOI 10.1007/978-94-017-8993-6

Library of Congress Control Number: 2014944543

Springer Dordrecht Heidelberg New York London

(C) Springer Science+Business Media Dordrecht 2014

This work is subject to copyright. All rights are reserved by the Publisher, whether the whole or part of the material is concerned, specifically the rights of translation, reprinting, reuse of illustrations, recitation, broadcasting, reproduction on microfilms or in any other physical way, and transmission or information storage and retrieval, electronic adaptation, computer software, or by similar or dissimilar methodology now known or hereafter developed. Exempted from this legal reservation are brief excerpts in connection with reviews or scholarly analysis or material supplied specifically for the purpose of being entered and executed on a computer system, for exclusive use by the purchaser of the work. Duplication of this publication or parts thereof is permitted only under the provisions of the Copyright Law of the Publisher's location, in its current version, and permission for use must always be obtained from Springer. Permissions for use may be obtained through RightsLink at the Copyright Clearance Center. Violations are liable to prosecution under the respective Copyright Law. The use of general descriptive names, registered names, trademarks, service marks, etc. in this publication does not imply, even in the absence of a specific statement, that such names are exempt from the relevant protective laws and regulations and therefore free for general use.

While the advice and information in this book are believed to be true and accurate at the date of publication, neither the authors nor the editors nor the publisher can accept any legal responsibility for any errors or omissions that may be made. The publisher makes no warranty, express or implied, with respect to the material contained herein.

Printed on acid-free paper

Springer is part of Springer Science+Business Media (www.springer.com) 
In memory of Hubert Curien (1924-2005), Professor at Pierre 85 Marie Curie University, Paris, and member of the French Academy of Sciences. A great administrator, Minister of Research in the French government, he shared his enthusiasm for crystallography with his students through the courses he gave throughout his career. 


\section{Foreword}

Apart from amorphous bodies such as glasses, the arrangement of ions, atoms and molecules in solid matter is regular and presents properties of symmetry. Crystals present both translational and orientational properties of symmetry, which govern their physical properties. It is because of their triple periodicity that crystals grow with a regular habit: quartz prisms, diamond octahedra or garnet rhomb-dodecahedra, and that they present planar cleavage faces, those of the cube for rock-salt, and those of the rhombohedron for Iceland spar. It is this same periodicity that is at the origin of the band structure of solids and conditions the properties of materials used in microelectronics. It is thanks to the redundancy of the information provided by the diffraction in phase of X-rays, neutrons or electrons, by all the molecular groups repeated by the translations of a crystal that one can determine their atomic structure. If one wishes to find the structure of a complex molecule such as a protein or a virus, one should therefore crystallize it. This is done systematically during the elaboration of drugs by the pharmaceutical industry. The orientational symmetry of crystals is present in all their properties, first of all in their external shape. But it is often because a particular element of symmetry is absent that a property may exist. It is because hydrated ammonium tartrate has no centre of symmetry that it is optically active, which Louis Pasteur associated to its external shape, right or left, thus discovering chirality (1848). It is because of symmetry considerations that the Curie brothers, Jacques and Pierre, looked for, and found, piezoelectricity in 1880. If one cools barium titanate below $120^{\circ} \mathrm{C}$, its structure is modified. Above, it is cubic holohedral, below, it is tetragonal, with as its only symmetry elements a four-fold axis and mirrors parallel to that axis. It is this symmetry which makes it possible for a spontaneous electric polarisation to appear, and for the crystal to become pyroand ferroelectric. Generally speaking, the properties associated to structural phase changes depend on the relations between symmetry groups and their subgroups.

The description of the symmetry operations of crystals and of the resulting physical properties is the aim of the present book. These operations are part of a complex system which was not grasped at once. It was a long way from Aristotle's elements to the concept of space lattice. The ancients were always fascinated by the regularity of six-sided prisms of rock-crystal, as noted by 
Pliny the Elder in his Natural History (77 A.D.). Kepler (1611), followed by Descartes (1637) and Bartholin (1661) tried to interpret the formation of sixcornered snowflakes by the agglomeration of six spherules round a seventh one. Kepler was the first to describe the primitive and face-centred cubic lattices and the hexagonal lattice, although he did not call them that way. Compact pile-ups were considered similarly by Hooke (1665) and Huygens (1678) to explain the shape of quartz and the cleavage rhombohedron of calcite, respectively. The constancy of interfacial angles was established in the seventeenth and the eighteenth centuries by Steno (1669), Carangeot (1780) and Romé de l'Isle (1783). At the end of the eighteenth century, Bergman (1773) for calcite and Haüy (1784) in a general way, showed that the external shape of crystal may be obtained by a regular and triply periodic pile-up of identical elemental parallelepipeds, now called unit cells, and called molécules intégrantes by Haüy. But Haüy confused the unit cell with its chemical content and did not accept the notion of isomorphism introduced by Mitscherlich (1819). His classification of crystals was based on their geometrical shape while that of the German School, led by Weiss (1817), was based on systems of symmetry axes. Haüy's main merit was nevertheless to have introduced the notion of three-dimensional periodicity which defines the crystalline medium and to have established the geometrical properties of lattice planes by means of the law of simple rational intercepts. The relations between symmetry and properties were established in steps all along the nineteenth century: the 7 crystal systems (Mohs, 1822), the notion of point lattice (Seeber, 1824), the 32 crystal classes (Frankenheim, 1826; Hessel, 1830), the notion of hemihedry (Delafosse, 1840), molecular chirality (Pasteur, 1848), the 14 Bravais lattices (1850), groups of motion including helicoidal motions, the 65 chiral groups (Sohncke 1865), and, finally, the 230 space groups (Fedorov, 1890; Schoenflies, 1891; Barlow, 1894). ${ }^{1}$

The description of physical properties in their geometrical form requires in general other mathematical tools, such as tensors, which were first introduced in elasticity by Voigt (1899) and Brillouin (1949). If one pulls a rod made of an isotropic material, its length increases and its cross-section decreases. A scalar is insufficient to describe the response of the material to an excitation and two constants are necessary to describe the resulting deformation. Similarly, the polarisation of an anisotropic dielectric can only be expressed by way of a tensor.

The book by Cécile Malgrange, Christian Ricolleau and Michel Schlenker is an extended translation of a first version in French by Cécile Malgrange, Christian Ricolleau and Françoise Lefaucheux. It comprises three parts of roughly equal importance: 1) a first part on crystal symmetry, symmetry operations, crystal lattices, point groups and space groups; 2) a second part on crystal anisotropy and tensors applied to elasticity, propagation of elastic waves in crystals and piezoelectricity; 3) a third one on the optical properties of crystals, polariza-

1. A. Authier, Early Days of X-Ray Crystallography (Oxford University Press, Oxford, 2013) 
tion, optical activity and electro-optical effects. The various types of chemical bonds are also described in a small chapter between the first and the second parts. Most chapters include an annex on a particular topic related to one of the points developed in the chapter, aimed at exciting the curiosity of the reader, and these complements are a real added value to the book. Exercises are also included, the solutions of which are given at the end of the book. They constitute a very valuable pedagogical tool. The whole of the book is written in a very clear and rigorous way.

The publication of a new, up-to-date, textbook of crystallography and crystal physics will be very welcome at a time when crystallography courses are disappearing in most universities throughout the world, although these disciplines represent basic knowledge compulsory for anyone doing material science, Earth science, biochemistry, solid state physics or chemistry.

André Authier

Professor Emeritus, Pierre 65 Marie Curie University, Paris Former President of the International Union of Crystallography Member of the German Academy of Sciences 


\section{Preface}

Crystallography constantly enjoys a new youth, thanks in particular to the spectacular progress in X-ray sources (synchrotron radiation from dedicated facilities and, very recently, free electron X-ray lasers), which make it possible to investigate an extremely broad range of materials. Its contribution is vital in many scientific and technological areas. It makes it possible, for example, to understand, and even to predict the properties of materials as diverse as high- $T_{c}$ superconductors, high performance magnets, or biological materials such as proteins. The pharmaceutical industry invests large funds to set up data banks of structures, determined in the crystallized state, of molecules which could become medicines, in order to correlate their configuration to their healing action. Crystallography also is very important in nanoscience, since some materials, when prepared in the form of nano-objects, take on crystal structures which do not exist in the bulk and which necessarily affect their physical and chemical properties. Geophysicists obtain major information about the Earth mantle by investigating crystallized materials under extreme temperature and pressure conditions. In technology, we must mention structural wonders such as thin film based artificial crystals (multilayers and superlattices) of semiconductors or magnetic materials. These have for several years been present in the giant or tunnel magnetoresistance read heads of the hard drives in all our computers.

On the other hand, understanding the relation between the structure and the macroscopic properties of crystals opens the way to the use of physical properties with many applications. Thus piezoelectricity, i.e. the production of an electrical voltage under the effect of stress, is the basis of our quartz watches and plays a central role in cell phones. Electro-optical effects, the change in refractive indices under the effect of an electric field, entail a change in light propagation in materials.

This book is mostly a translation of Symétrie et propriétés physiques des cristaux, published by EDP Sciences, Orsay, France in 2011. It provides the fundamentals underpinning all these research and application areas.

In the first part, it describes in a clear and thorough way the periodicity of crystals and their symmetry, both at the microscopic level (space groups) and at the macroscopic level (point groups). It includes a presentation of the crystallography of two-dimensional systems such as surfaces and interfaces. 
The second part is dedicated to various physical properties of crystals. They are described in the tensor formalism which is natural for them, emphasizing their link with crystal symmetry. We show in particular how a given property is allowed or forbidden by various crystal symmetries, and how the coefficients of the characteristic tensor are related for the various point groups. We deal not only with basic properties such as elasticity and birefringence, but also with properties which are less often dealt with, such as piezoelectricity, optical activity or the electro-optical effects.

Each chapter starts with a short presentation of its contents, and almost all chapters are complemented by exercises through which the notions introduced can be concretely applied, and thus mastered. For these exercises to be a real studying tool, the solutions are explicitly given as a final separate Chapter 20 .

Many of the chapters also include a brief complement, intended to give a glimpse into an area related to that of the chapter and to whet the reader's scientific appetite, for example on how and what for crystals are grown, with what probes crystal structures can be determined, etc.

This book originates in courses we gave at Paris Diderot University and at Grenoble Institute of Technology, France. It is aimed, with no special prerequisite, at students in crystallography, physics and engineering (especially materials science and electrical engineering) curricula, and at $\mathrm{PhD}$ students. It should also be a reference book for researchers and engineers involved, in their daily activity, in the properties of crystals.

The authors sincerely thank the five members of the Advisory Editorial Committee for their very valuable suggestions, the vast majority of which were followed. They are also very grateful to Professor André Authier for writing the Foreword, which actually is a very informative chapter on the history of crystallography.

The authors intend to correct the mistakes and typos which will, in spite of their efforts, have remained in this first edition. Errata sheets will be provided at

https://grenoble-sciences.ujf-grenoble.fr/ouvrage/symmetry-crystals Comments from readers are very welcome. Please write to

symmetry-book@impmc.upmc.fr

Cécile Malgrange, Christian Ricolleau, Michel Schlenker. 


\title{
Table of symbols
}

\author{
$\mathbf{a}, \mathbf{b}, \mathbf{c}$ \\ Basis vectors of the unit cell in direct space \\ $a, b, c$ \\ Lengths of the basis vectors of the unit cell in direct space \\ $\mathbf{a}^{*}, \mathbf{b}^{*}, \mathbf{c}^{*}$ \\ Basis vectors of the reciprocal unit cell \\ $a^{*}, b^{*}, c^{*}$ \\ Lengths of the basis vectors of the reciprocal unit cell \\ $v_{0}$ \\ $v_{0}^{*}$ \\ Volume of the direct unit cell \\ Volume of the reciprocal unit cell \\ $[u v w]$ \\ Lattice row parallel to the vector $u \mathbf{a}+v \mathbf{b}+w \mathbf{c}$ \\ $<u v w>$ \\ Set of lattice rows equivalent to $[u v w]$ through all the sym- \\ metry operations of the crystal group \\ $(h k l)$ \\ Family of lattice planes with Miller indices $h, k$ and $l$ \\ (hkil) \\ $\{h k l\}$ \\ $\{h k i l\}$ \\ $d_{h k l}$ \\ Family of lattice planes with Miller indices $h, k, i$ and $l$ for \\ a hexagonal lattice \\ Set of lattice planes equivalent to $(h k l)$ through the symme- \\ try operations of the crystal group \\ Set of lattice planes equivalent to ( $h k i l)$ through the symme- \\ try operations of the crystal group, for a hexagonal lattice \\ Distance between two neighboring $(h k l)$ lattice planes, i.e. \\ the lattice spacing of family $(h k l)$ \\ $\mathbf{e}_{1}, \mathbf{e}_{2}, \mathbf{e}_{3}$ \\ Basis vectors of an orthonormal frame \\ $\left\{M_{i j}\right\}$ \\ $\left\{a_{i j}\right\}=A$ \\ The matrix for which $M_{i j}$ is the coefficient located on the \\ $i$-th row and the $j$-th column \\ $\stackrel{\mathbf{V}}{\mathbf{V}}$ \\ Transition matrix for going over from an orthonormal frame \\ to another one \\ Polar vector \\ Axial vector \\ $[T]$ \\ Tensor \\ $T_{i j}$ \\ Components, in a given orthonormal axis system, of a rank-2 \\ tensor $[T]$. The associated matrix is noted $T$. \\ $T_{i j k} \quad$ Components, in a given orthonormal axis system, of a rank-3 \\ tensor $[T]$
}


$e_{i j} \quad$ Components of the tensor of displacement gradients, or distorsion tensor, $[e]$

$\mathrm{T} \quad$ The set of the crystal lattice translations

$G_{s} \quad$ Space group

$G_{p} \quad$ Point group

$[S], S_{i j}$

Strain tensor, and its components

[T], $T_{i j}$

Stress tensor, and its components

$S_{\alpha}, T_{\alpha}$

Contracted notation (Voigt's notation) for the tensors $[S]$ and $[T] . \alpha$ ranges from 1 to 6 , so that the components of these tensors form column vectors with 6 rows.

$[s], s_{i j k l} \quad$ Compliance tensor, and its components

$[c], c_{i j k l} \quad$ Stiffness tensor, and its components

$\Theta$

Temperature

$\mathcal{S}$

Entropy

E

Electric field

D

Electric induction

$\mathbf{P}$

Polarization

$[\varepsilon], \varepsilon_{i j}$

$\varepsilon_{0}$

$[\eta], \eta_{i j}$

$\delta_{i j}$

Relative permittivity (or dielectric constant) tensor, and its components

Permittivity (or dielectric constant) of vacuum

$\delta_{i j k}$

Electric impermeability tensor, and its components

Kronecker symbol

Levi-Civita, or permutation, tensor

$[G], G_{i j}$

Gyration tensor, and its components

$[d], d_{i j k}$

Piezoelectric tensor, and its components

$[r], r_{i j k}$

Linear electro-optic tensor, or Pockels tensor, and its components

$[z], z_{i j k l} \quad$ Quadratic electro-optic tensor, or Kerr tensor, and its components

$[p], p_{i j k l} \quad$ Elasto-optic tensor and its components

$[\pi], \pi_{i j k l} \quad$ Piezo-optic tensor and its components 


\section{Contents}

Chapter 1 - Introduction $\quad \mathbf{1}$

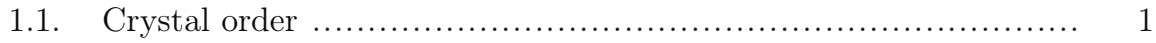

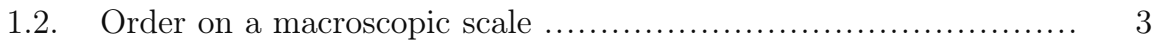

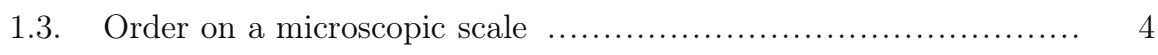

1.4. Basic assumptions of geometrical crystallography $\ldots \ldots \ldots \ldots \ldots \ldots . . \ldots$

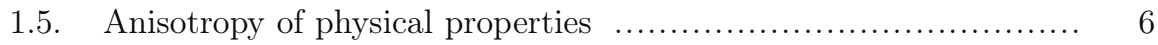

1.6. Remarks on the scope of this book : topics left out .............. 7

Complement 1C. Crystal growth .................................. 8

1C.1. Natural growth of single crystals ...................... 8

1C.2. Crystal growth for scientific purposes ................... 8

1C.3. Industrial crystal growth ........................... 9

1C.4. Growth of single-crystal thin films $\ldots \ldots \ldots \ldots \ldots \ldots \ldots \ldots . . \ldots$

Further reading $\ldots \ldots \ldots \ldots \ldots \ldots \ldots \ldots \ldots \ldots \ldots \ldots \ldots \ldots \ldots \ldots \ldots, 10$

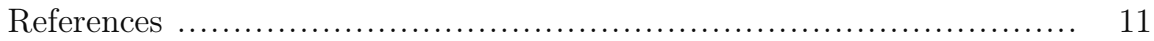

Chapter 2 - Symmetry operations $\quad \mathbf{1 3}$

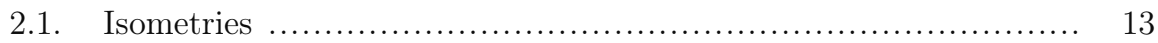

2.2. Symmetry operations. Symmetry elements ..................... 14

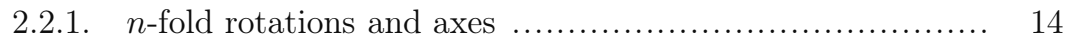

2.2.2. $n$-fold rotoinversions and $n$-fold rotoinversion axes,

2.2.3. Translations ............................................. 16

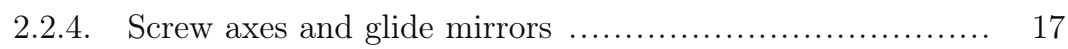

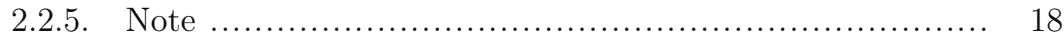

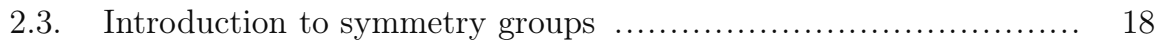

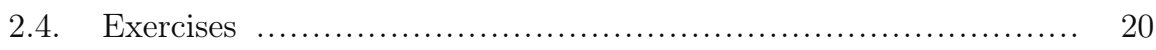

Chapter 3 - Crystal lattices $\quad 23$

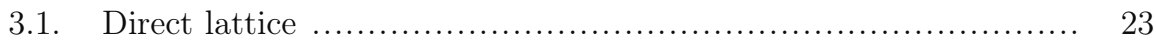

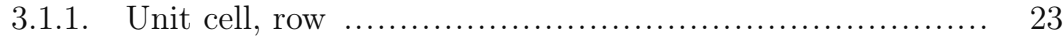

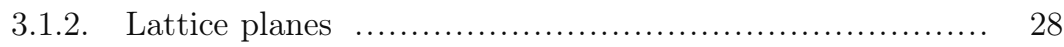


3.1.3. Wigner-Seitz cell ................................. 32

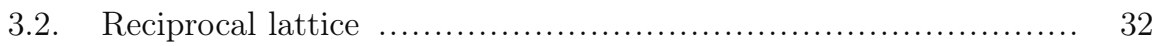

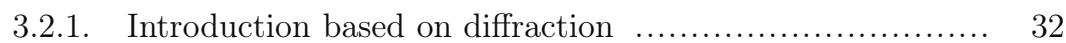

3.2.2. Alternative definition of the reciprocal lattice $\ldots \ldots \ldots \ldots \ldots .34$

3.2.3. Properties of the reciprocal lattice ..................... 35

3.2.4. Crystallographic calculations ......................... 37

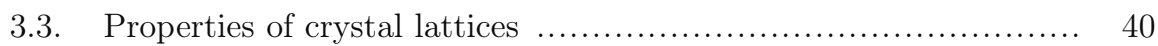

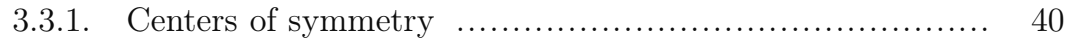

3.3.2. $n$-fold and $\bar{n}$-fold axes consistent with the crystalline state 40

3.3.3. The direct lattice and the reciprocal lattice have the same symmetry elements $\ldots \ldots \ldots \ldots \ldots \ldots \ldots \ldots \ldots \ldots \ldots, 41$

3.3.4. Geometrical relation between the symmetry axes and the crystal lattice $\ldots \ldots \ldots \ldots \ldots \ldots \ldots \ldots \ldots \ldots \ldots \ldots, 42$

3.3.5. The lattice is at least as symmetrical as the crystal ...... 43

3.4. Crystal systems .............................................. 43

3.4.1. Two-dimensional crystal systems $\ldots \ldots \ldots \ldots \ldots \ldots \ldots \ldots \ldots . \ldots \ldots$

3.4.2. Three-dimensional crystal systems .................... 44

3.5. Examples of reciprocal lattices ............................. 47

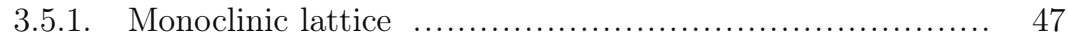

3.5.2. Orthorhombic, tetragonal and cubic lattices ............. 47

3.6. Hexagonal lattice and rhombohedral lattice .................... 48

3.6.1. Hexagonal lattice ................................... 48

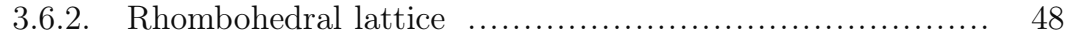

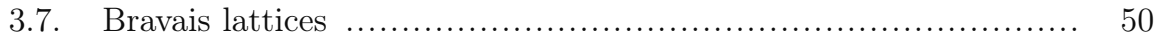

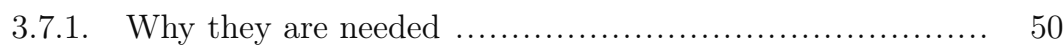

3.7.2. The fourteen Bravais lattices .......................... 52

3.7.3. Reciprocal lattices of the non-primitive lattices .......... 53

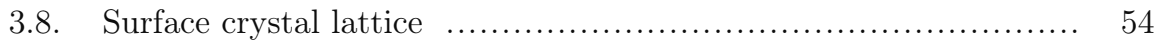

3.8.1. Cut surface .......................................... 55

3.8.2. Real surface ...................................... 55

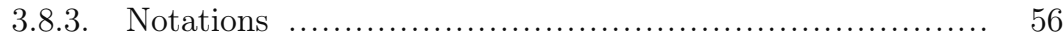

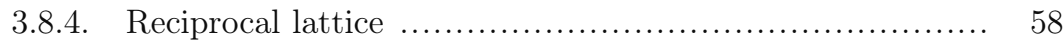

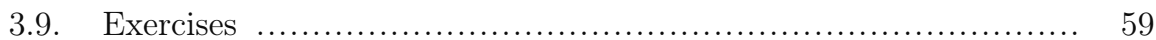

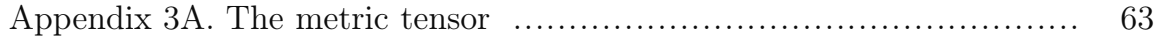

3A.1. Definition ......................................... 63

3A.2. Volume of the unit cell .................................. 63

3A.3. Product of the matrices associated to the direct and reciprocal metric tensors $\ldots \ldots \ldots \ldots \ldots \ldots \ldots \ldots \ldots \ldots \ldots, 64$

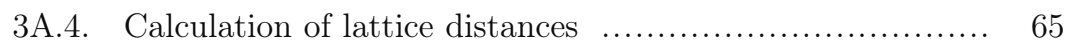

3A.5. Applications ........................................ 65 


\section{Chapter 4 - Relationship between space groups} and point groups

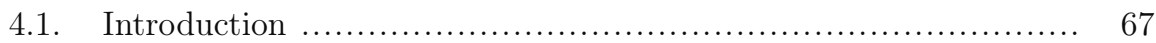

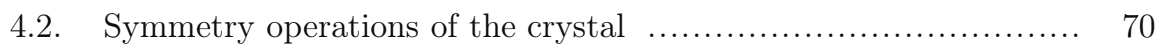

4.2.1. Change in origin .................................... 70

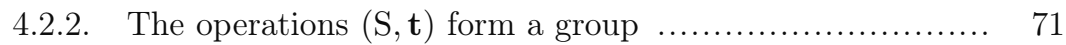

4.2.3. The lattice translations form an invariant subgroup of the group of symmetry operations of the crystal ........ 72

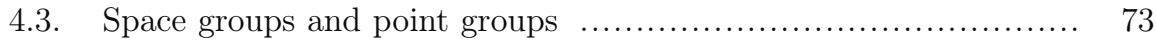

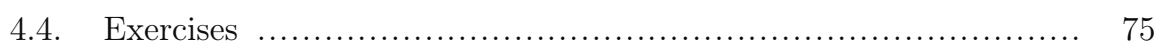

Complement 4C. Probes used for crystal structure determination ....... 76

4C.1. Possible probes and criteria for choice ................. 76

4 C.2. X-rays .......................................... 76

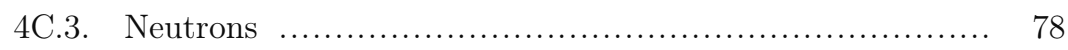

4C.4. Electrons ............................................. 79

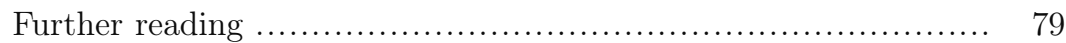

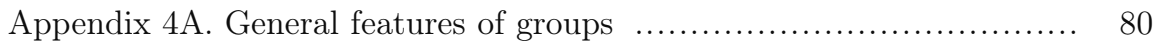

Chapter 5 - Point groups $\quad 83$

5.1. Introduction ................................................. 83

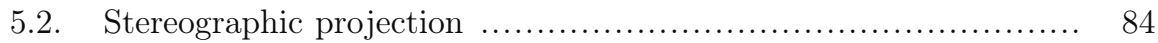

5.2.1. Definition ........................................ 84

5.2.2. Examples ......................................... 86

5.2.3. Application to rotoinversion axes or $\bar{n}$-fold axes ......... 88

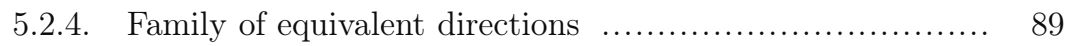

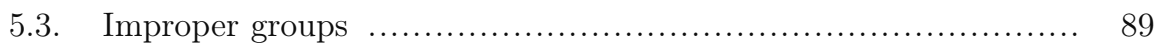

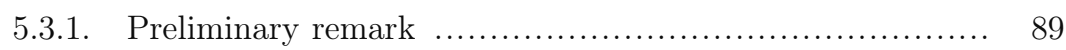

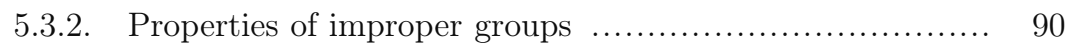

5.4. Enumeration of the proper point groups $\ldots \ldots \ldots \ldots \ldots \ldots \ldots \ldots \ldots . \ldots 1$

5.4.1. Preamble .......................................... 91

5.4.2. Groups containing only the symmetry operations associated to an axis $\mathrm{A}_{n}$ (cyclic groups) $\ldots \ldots \ldots \ldots \ldots \ldots . \ldots 2$

5.4.3. Groups containing the symmetry operations associated to an axis $A_{n}$ and to a perpendicular axis $A_{2}$,

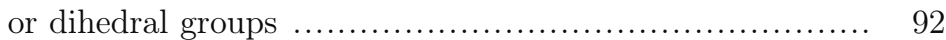

5.4.4. Cubic proper groups ................................ 93

5.5. Enumeration of the improper point groups .................... 94

5.5.1. Improper groups containing inversion .................. 94

5.5.2. Improper groups which do not feature inversion .......... 97

5.6. Classification of the point groups f.......................... 101 


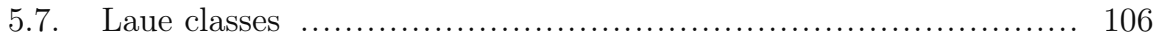

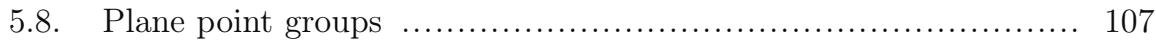

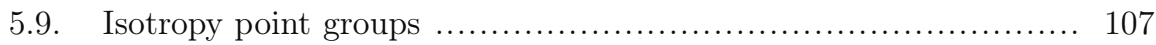

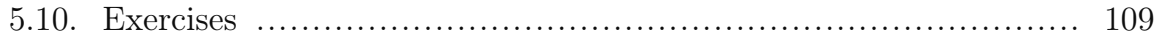

Appendix 5A. Complements on the stereographic projection ............ 111

5A.1. Stereographic projection of the transform of a given direction through the symmetry operations associated to various symmetry elements $\ldots \ldots \ldots \ldots \ldots \ldots \ldots \ldots \ldots \ldots \ldots \ldots \ldots 11$

5A.2. Stereographic projections of the symmetry elements of a cube ........................................... 112

$\begin{array}{lr}\text { Chapter } 6 \text { - Bravais lattices } & 117\end{array}$

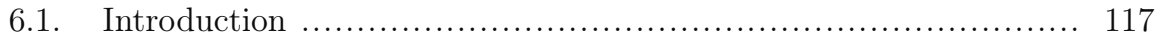

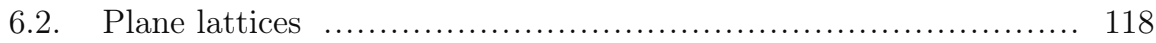

6.2.1. Group 1 ....................................... 118

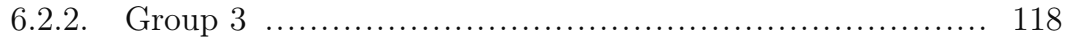

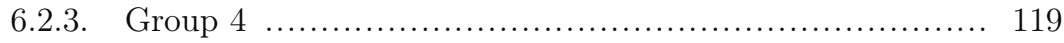

6.2.4. Group $\mathrm{m}$.......................................... 119

6.2.5. Conclusion .......................................... 120

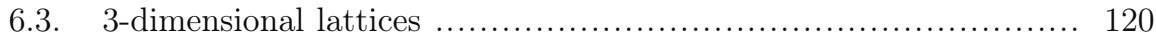

6.3.1. Group 1 ....................................... 120

6.3.2. Group 2 ........................................ 120

6.3.3. Group 3 ......................................... 122

6.3.4. Group 4 ........................................ 124

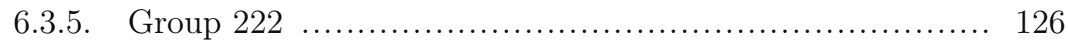

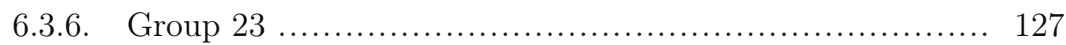

$\begin{array}{lr}\text { Chapter } 7 \text { - Space groups } & 129\end{array}$

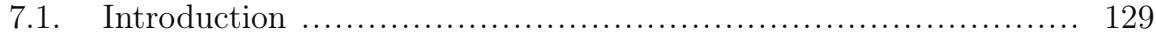

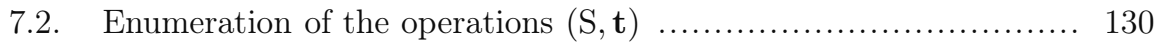

7.2.1. $\mathrm{S}$ is a rotation. Definition of screw axes ................. 131

7.2.2. $\mathrm{S}$ is a rotoinversion, noted $\overline{\mathrm{S}}$. Definition of glide mirrors .. 134

7.2.3. Product of a symmetry operation and a translation ...... 136

7.3. Enumeration of space groups .............................. 139

7.3.1. Symmorphic space groups ......................... 139

7.3.2. Non-symmorphic space groups ...................... 142

7.3.3. International Tables for Crystallography ................. 146

7.4. Nomenclature ............................................ 149

7.5. Examples: space groups of some structures $\ldots \ldots \ldots \ldots \ldots \ldots \ldots \ldots \ldots \ldots \ldots$

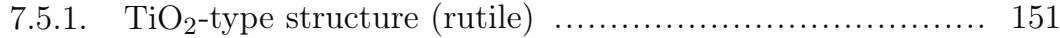


7.5.2. Metals with close-packed hexagonal structure ............ 152

7.5.3. Structure of diamond ................................. 154

7.6. Exercises ....................................................... 155

Complement 7C. Structure determination: outline $\ldots \ldots \ldots \ldots \ldots \ldots \ldots \ldots \ldots$

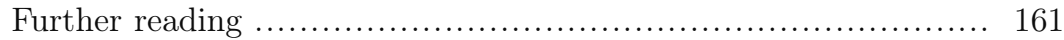

References ......................................................... 162

Chapter 8 - Chemical bonds and crystal structures 163

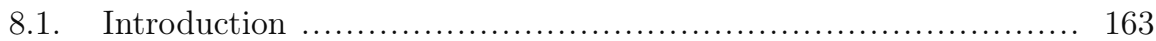

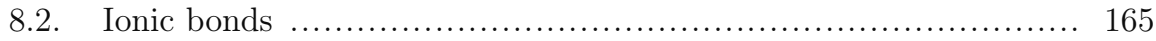

8.2.1. Nature and properties ............................... 165

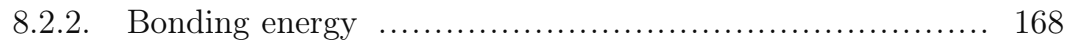

8.2.3. Ionic structures with formula AX ...................... 169

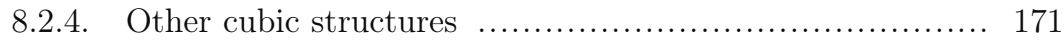

8.3. Covalent bonds ............................................... 173

8.3.1. Nature of covalent bonds .............................. 173

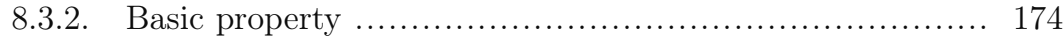

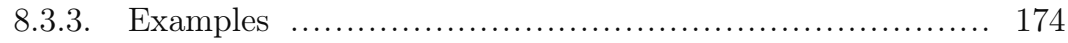

8.3.4. Conclusion ......................................... 176

8.4. Van der Waals bonds, or molecular bonds ....................... 177

8.4.1. Nature and properties .............................. 177

8.4.2. Example ......................................... 177

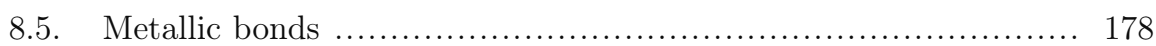

8.5.1. Nature and properties ............................... 178

8.5.2. Examples ...................................... 178

8.6. Remarks and conclusions f................................ 180

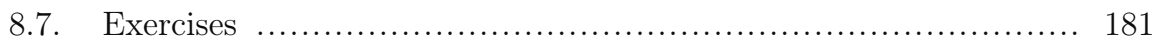

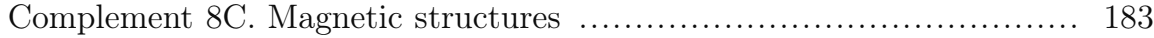

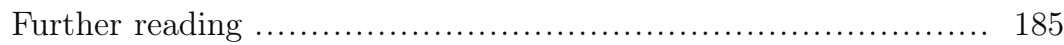

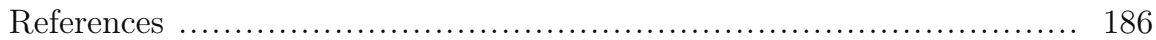

$\begin{array}{lr}\text { Chapter } 9 \text { - Crystal anisotropy and tensors } & 187\end{array}$

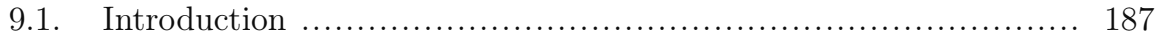

9.2. Anisotropic continuous medium .............................. 188

9.3. Representing a physical quantity by a tensor $\ldots \ldots \ldots \ldots \ldots \ldots \ldots \ldots . \ldots 189$

9.3.1. Example: electrical conductivity ....................... 189

9.3.2. A refresher on orthonormal frame changes $\ldots \ldots \ldots \ldots \ldots \ldots .191$

9.3.3. Application to electrical conductivity .................. 193

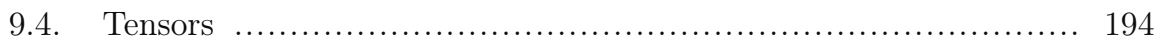

9.4.1. Definition of a tensor ................................ 194 


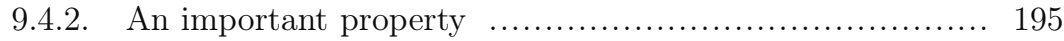

9.4.3. Field tensors and material tensors ..................... 196

9.5. Symmetry properties of tensors ............................ 197

9.5.1. Internal symmetry. Symmetric and antisymmetric tensors 197

9.5.2. External symmetry of material tensors.

Curie's and Neumann's principles ..................... 197

9.6. Reduction in the number of independent coefficients

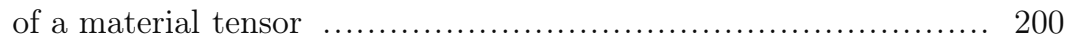

9.6.1. Method using the transformation matrix ................ 200

9.6.2. "Direct inspection" method ........................... 201

9.6.3. Special case: central symmetry (inversion symmetry) ..... 202

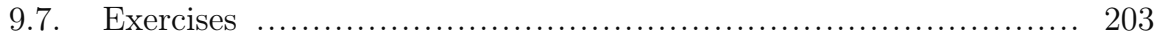

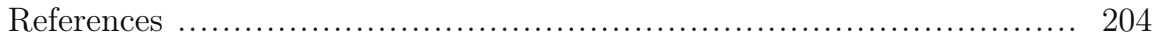

Chapter 10 - Second-rank tensors $\quad 205$

10.1. Introduction ............................................ 205

10.1.1. Symmetric and antisymmetric tensors .................. 205

10.1.2. Matrix form of second-rank tensors f.................. 206

10.1.3. Trace ........................................... 206

10.2. Representative quadric for a symmetric rank-2 tensor ........... 207

10.2.1. Characteristic surface .............................. 207

10.2.2. Principal axes and principal coefficients ................ 207

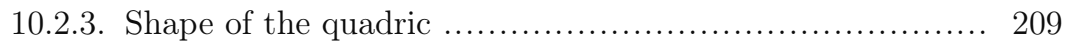

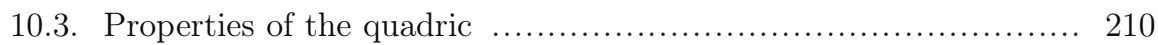

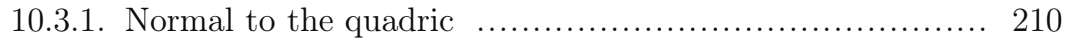

10.3.2. Length and physical meaning of the radius vector ........ 211

10.3.3. Intensity of a physical property in a given direction ....... 211

10.4. Geometrical determination of the principal axes and principal

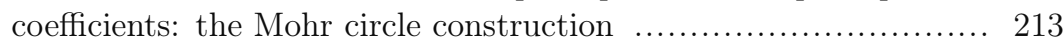

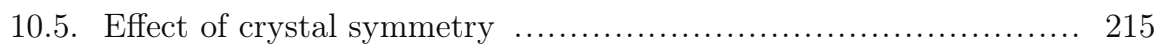

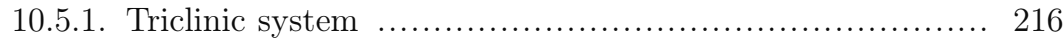

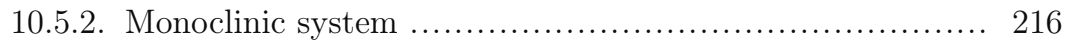

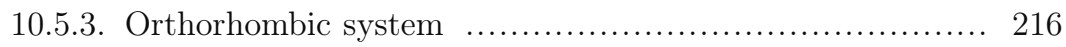

10.5.4. Uniaxial systems: rhombohedral, trigonal and hexagonal . 217

10.5.5. Cubic system .................................... 217

10.6. Axial vectors, or antisymmetric rank-2 tensors $\ldots \ldots \ldots \ldots \ldots \ldots \ldots . \ldots \ldots$

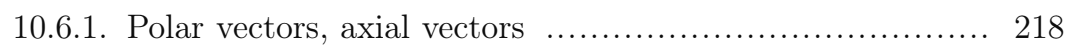

10.6.2. Example of an axial vector: the cross product ........... 220

10.7. Exercises .............................................. 222 
Chapter 11 - The stress tensor $\quad 225$

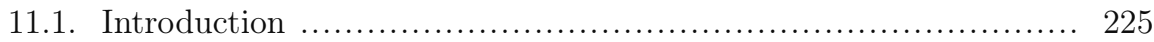

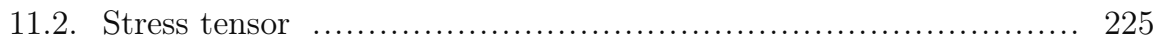

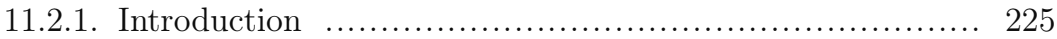

11.2.2. Definition of the stress tensor $\ldots \ldots \ldots \ldots \ldots \ldots \ldots \ldots \ldots \ldots \ldots \ldots \ldots \ldots \ldots \ldots$

11.2.3. Normal stress and shear stress ........................ 229

11.3. Basic relation .............................................. 230

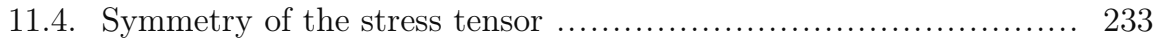

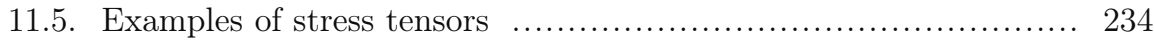

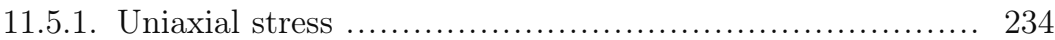

11.5.2. Pure shear ........................................... 235

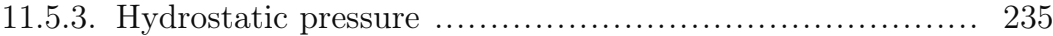

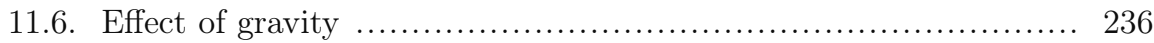

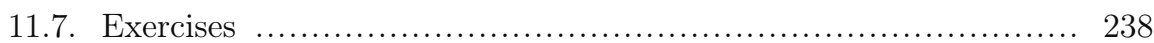

References ......................................................... 240

Chapter 12 - Deformation of a solid. The strain tensor $\quad 241$

12.1. Distortion tensor (displacement gradient tensor) ............... 241

12.1.1. Definition .......................................... 241

12.1.2. Physical meaning of components $e_{i j} \quad \ldots \ldots \ldots \ldots \ldots \ldots \ldots . \ldots 243$

12.2. Decomposition of the distortion tensor

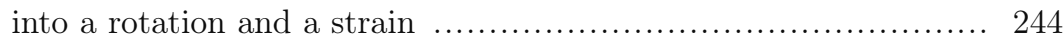

12.2.1. Introduction using a simple example ................... 244

12.2.2. Expressing the distortion associated to small rotations .... 245

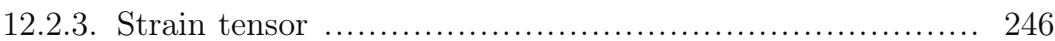

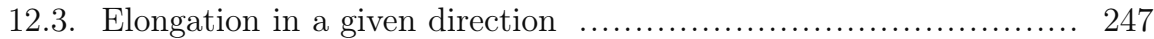

12.4. Volume expansion (volumetric strain) ......................... 248

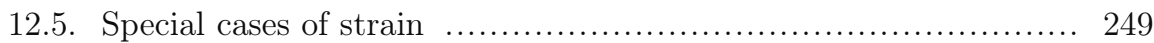

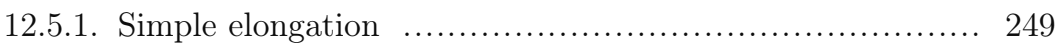

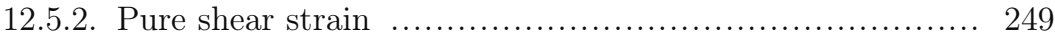

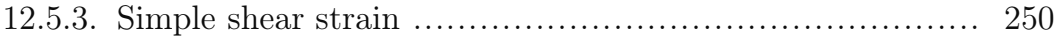

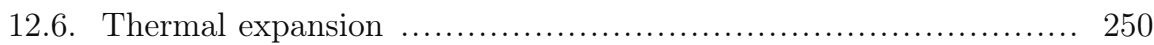

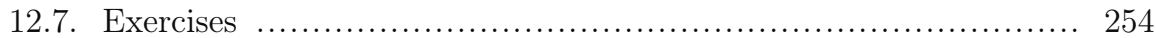

Chapter 13 - Elasticity $\quad 259$

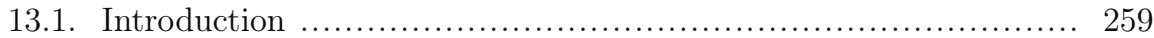

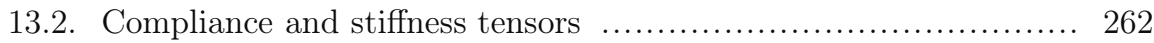

13.2.1. Generalized Hooke's law .................................... 262

13.2.2. Symmetry of the compliance and stiffness tensors ......... 263 
13.3. Contracted notation (Voigt's notation) ...................... 264

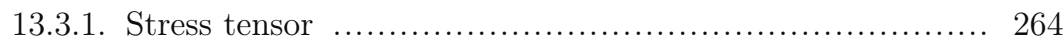

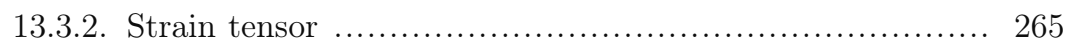

13.3.3. Compliance tensor and stiffness tensor ................. 265

13.3.4. Relation between the compliance and stiffness tensors .... 268

13.4. Energy of a strained solid ................................. 268

13.5. Effect of crystal symmetry on the form of the elastic tensor ....... 271

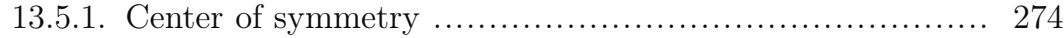

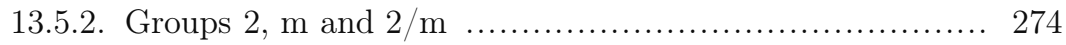

13.5.3. Groups $222, \mathrm{mmm}$ and $\mathrm{mm} 2$...................... 274

13.5.4. Groups 422, $4 \mathrm{~mm}$ and $4 / \mathrm{mmm}$..................... 275

13.5.5. Cubic system ..................................... 276

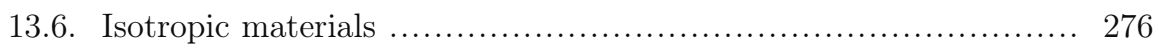

13.6.1. Expressing components $s_{\alpha \beta}$ as a function of $E$ and $\nu \ldots \ldots 278$

13.6.2. Stiffness components. Lamé coefficients .................. 279

13.7. Representative surface for Young's modulus $\ldots \ldots \ldots \ldots \ldots \ldots \ldots \ldots . . \ldots 279$

13.8. Compressibility ......................................... 281

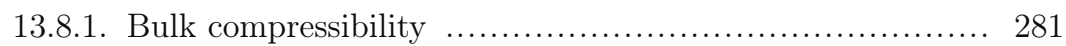

13.8.2. Linear compressibility of a bar ........................ 282

13.9. Notes on non-uniform stresses and strains ................... 283

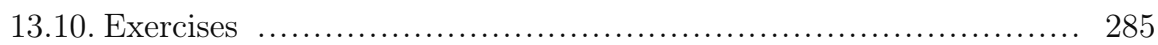

Complement 13C. Plastic deformation and crystal defects $\ldots \ldots \ldots \ldots \ldots \ldots .288$

Further reading ......................................... 290

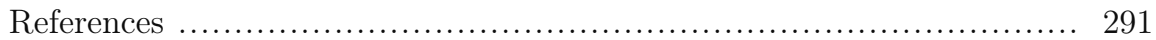

Chapter 14 - Elastic waves in crystals $\quad 293$

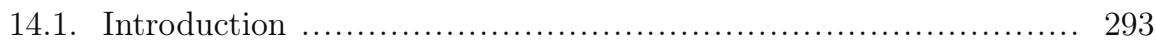

14.2. Plane elastic waves ........................................ 294

14.3. Application to a cubic crystal ............................... 297

14.3.1. Propagation of a plane wave along direction [100] ........ 298

14.3.2. Propagation along direction $[110]$...................... 299

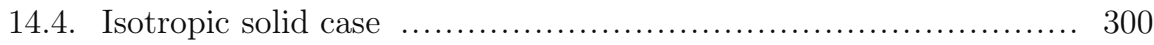

14.5. Microscopic approach - Crystal lattice dynamics ................ 301

14.5.1. Linear chain of identical atoms ......................... 301

14.5.2. Linear chain with two different atoms ..................... 305

14.5.3. Extension to the real crystal case ...................... 308

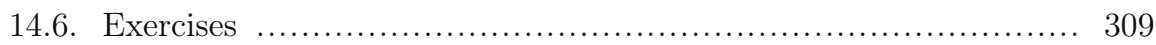


Chapter 15 - Crystal thermodynamics. Piezoelectricity

15.1. Crystal thermodynamics ................................... 312

15.1.1. Conjugate variables ................................ 312

15.1.2. Independent variables .............................. 314

15.1.3. Principal effects $v s$ crossed effects ..................... 317

15.1.4. Summary of the various effects $\ldots \ldots \ldots \ldots \ldots \ldots \ldots \ldots \ldots \ldots . \ldots \ldots$

15.1.5. Condensed representation of the physical property matrix 320

15.2. Pyroelectricity. Pyroelectric crystals ........................... 321

15.3. Piezoelectric crystals .................................... 322

15.3.1. Direct effect and inverse effect ....................... 322

15.3.2. Piezoelectric tensor $[d]$ and its two-subscript notation .... 323

15.3.3. Effect of crystal symmetry on the form of the tensor ...... 324

15.3.4. Longitudinal piezoelectricity surface ................... 328

15.3.5. Other forms of piezoelectric coefficients ................ 329

15.3.6. Applications ......................................... 331

15.4. Principal and crossed effects under various conditions ........... 332

15.5. Exercises ................................................ 334

Complement 15C. Electrostriction and magnetostriction ................ 337

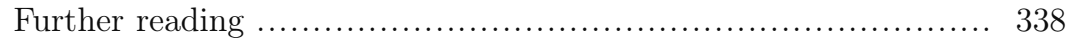

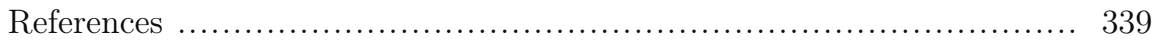

Chapter 16 - Light propagation in crystals $\quad 341$

16.1. Maxwell's equations ..................................... 341

16.2. Light propagation in an isotropic material ...................... 342

16.3. Sinusoidal waves that are solutions of Maxwell's equations ....... 343

16.4. Plane monochromatic wave in an anisotropic material ............ 345

16.4.1. Basic equation ......................................... 345

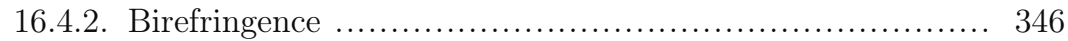

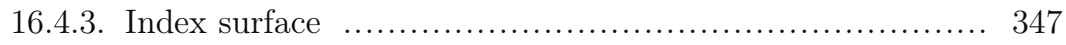

16.4.4. Index ellipsoid ...................................... 349

16.4.5. Determining the induction vectors .................... 350

16.4.6. Direction of energy propagation ........................ 353

16.5. Refraction of a plane wave at the boundary between two materials 354

16.5.1. The wave-vectors follow the Snell-Descartes law .......... 354

16.5.2. Application to uniaxial materials ........................ 357

16.6. Conclusion ............................................... 358

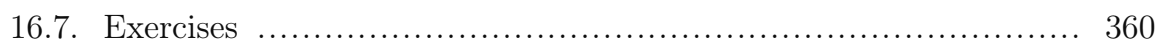


Appendix 16A. Wave surface (or ray surface) and Huygens' construction 364

16A.1. Wave surface (or ray surface) ...................... 364

16A.2. Huygens' construction ............................. 367

Chapter 17 - Polarization of light by crystals $\quad 369$

17.1. Polarization state of an electromagnetic wave ................... 369

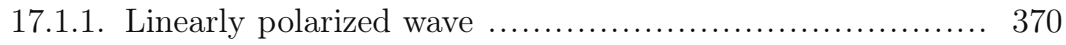

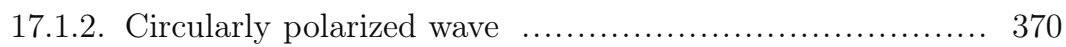

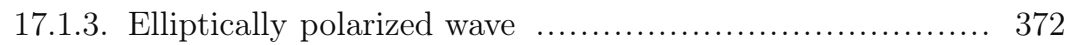

17.1.4. Natural light ..................................... 372

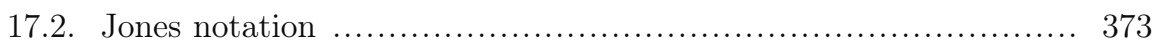

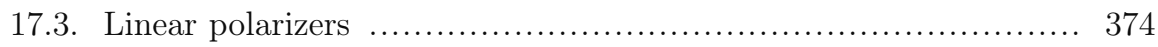

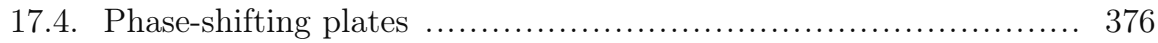

17.4.1. Half-wave plates $\ldots \ldots \ldots \ldots \ldots \ldots \ldots \ldots \ldots \ldots \ldots \ldots \ldots \ldots \ldots \ldots \ldots$

17.4.2. Quarter-wave plates .............................. 378

17.5. Partially polarized waves and Stokes parameters ................. 379

17.6. Exercises ................................................ 382

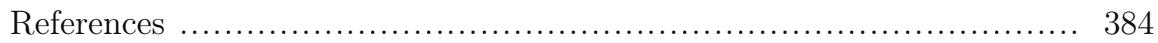

Chapter 18 - Rotatory power and optical activity 385

18.1. Definition of the rotatory power of a material .................. 385

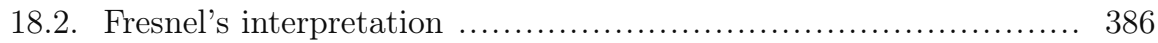

18.3. Interpretation of rotatory power through the influence
of the local environment $\ldots \ldots \ldots \ldots \ldots \ldots \ldots \ldots \ldots \ldots \ldots \ldots \ldots \ldots \ldots \ldots \ldots \ldots \ldots \ldots \ldots \ldots \ldots$

18.3.1. Effect of spatial dispersion $\ldots \ldots \ldots \ldots \ldots \ldots \ldots \ldots \ldots \ldots \ldots \ldots \ldots \ldots \ldots$

18.3.2. Wave propagation in optically active crystals ........... 390

18.4. Effect of crystal symmetry on the gyrotropy tensor ............... 393

18.4.1. Centrosymmetric groups $\ldots \ldots \ldots \ldots \ldots \ldots \ldots \ldots \ldots \ldots \ldots \ldots \ldots \ldots \ldots \ldots \ldots \ldots$

18.4.2. Non-centrosymmetric groups $\ldots \ldots \ldots \ldots \ldots \ldots \ldots \ldots \ldots \ldots \ldots . \ldots \ldots 4$

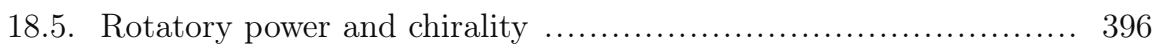

18.6. Absorption and conclusion ................................ 400

Complement 18C. Magnetic optical rotation .......................... 401

Further reading ............................................ 402

Appendix 18A. Axial tensors, or pseudo-tensors ...................... 403

18A.1. Definition of axial tensors, or pseudo-tensors $\ldots . \ldots \ldots \ldots \ldots . . \ldots 4$

18A.2. Levi-Civita tensor, or permutation tensor ................ 403

18A.3. The gyrotropy tensor $[G]$ is a rank-2 axial tensor ......... 404

18A.4. Relation between tensors $[G]$ and $[\beta] \ldots \ldots \ldots \ldots \ldots \ldots \ldots . \ldots . \ldots . \ldots . \ldots . \ldots$

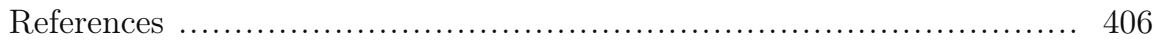


Chapter 19 - Electro-optical and elasto-optical effects $\quad 407$

19.1. Introduction ............................................. 407

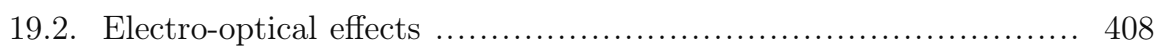

19.2.1. Linear electro-optical effect, or Pockels effect .............. 408

19.2.2. Applications of the linear electro-optical effect ............ 415

19.2.3. Quadratic electro-optical effect, or Kerr effect ............ 420

19.3. Elasto-optical effects ...................................... 424

19.3.1. Definition ............................................. 424

19.3.2. Application to the acousto-optical effects ............... 427

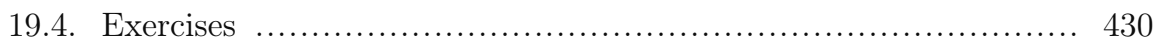

Complement 19C. Frequency doubling, or Second Harmonic Generation 433

Further reading ........................................... 434

Chapter 20 - Solutions to the exercises $\quad 435$

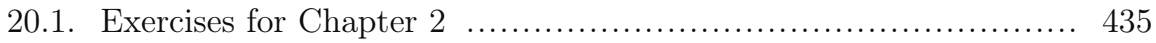

20.2. Exercises for Chapter 3 ..................................... 436

20.3. Exercises for Chapter 4 ................................... 444

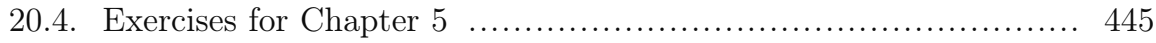

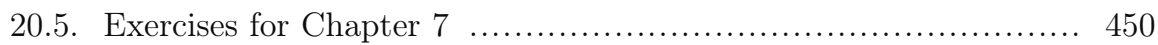

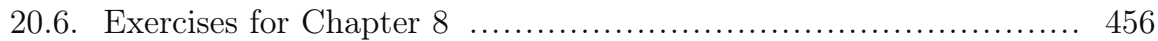

20.7. Exercises for Chapter 9 ................................... 458

20.8. Exercises for Chapter 10 .................................. 459

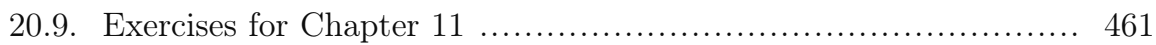

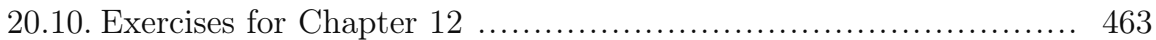

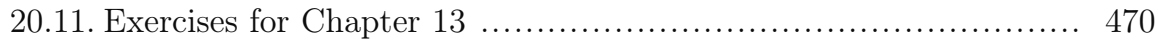

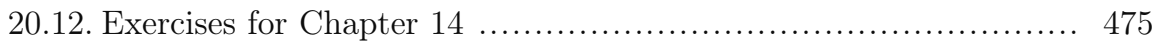

20.13. Exercises for Chapter 15 ...................................... 479

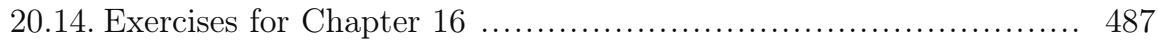

20.15. Exercises for Chapter 17 .................................. 492

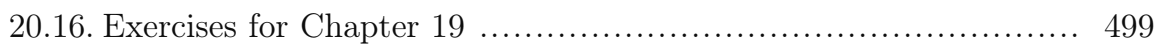

$\begin{array}{lr}\text { General references } & 507\end{array}$

$\begin{array}{ll}\text { Index } & 511\end{array}$ 